\title{
Ion-implantation induced nano distortion layer and its influence on nonlinear optical properties of $\mathrm{ZnO}$ single crystals
}

\author{
C. C. Zheng, ${ }^{1}$ S. J. Xu ${ }^{1, \text { a) }}$ J. Q. Ning, ${ }^{1}$ Y. N. Chen, ${ }^{1, b)}$ X. H. Lu, ${ }^{2}$ C.-C. Ling, ${ }^{2}$ C. M. Che, ${ }^{3}$ \\ G. Y. Gao, ${ }^{4}$ J. H. Hao, ${ }^{4}$ G. Brauer, ${ }^{5}$ and W. Anwand ${ }^{5}$ \\ ${ }^{1}$ Department of Physics, HKU-CAS Joint Laboratory on New Materials, The University of Hong Kong, \\ Pokfulam Road, Hong Kong, China \\ ${ }^{2}$ Department of Physics, The University of Hong Kong, Pokfulam Road, Hong Kong, China \\ ${ }^{3}$ Department of Chemistry, Institute of Molecular Functional Materials, HKU-CAS Joint Laboratory on \\ New Materials, The University of Hong Kong, Pokfulam Road, Hong Kong, China \\ ${ }^{4}$ Department of Applied Physics, The Hong Kong Polytechnic University, Hung Hom, Hong Kong, China \\ ${ }^{5}$ Institut für Strahlenphysik, Helmholtz-Zentrum Dresden-Rossendorf, Postfach 510119, D-01314 Dresden, \\ Germany
}

(Received 11 July 2011; accepted 31 August 2011; published online 19 October 2011)

\begin{abstract}
Second harmonic generation (SHG) and X-ray diffraction rocking curves of high-quality $\mathrm{ZnO}$ single crystals implanted by different ions $(\mathrm{He}, \mathrm{Cu}$, and $\mathrm{Zn})$ were investigated. Interestingly, it was found that both $\mathrm{He}$ - and $\mathrm{Zn}$-implanted samples show a convinced increment in SHG efficiency while the $\mathrm{Cu}$-implanted one does not. X-ray diffraction rocking curves of the samples show satellite structures, and the simulations firmly reveal the formation of quasi-interfaces inside He- and $\mathrm{Zn}$-implanted crystals. These quasi-interfaces lead to SHG improvement in the two samples. Polarization dependence of SHG of the samples on the excitation light also evidences this conclusion. (C) 2011 American Institute of Physics. [doi:10.1063/1.3651379]
\end{abstract}

\section{INTRODUCTION}

In recent years, $\mathrm{ZnO}$ has attracted a renewed interest because of its potential applications in fabricating ultraviolet optoelectronic devices, laser diodes, solar cells, spintronics, and nonlinear optical devices. ${ }^{1,2}$ In order to explore these potential applications, different treatments have been introduced to modify and manipulate properties of $\mathrm{ZnO} .{ }^{3}$ In particular, as a well-established technique for modifying properties of semiconductors, ion-implantation has been used to manipulate the electric and thermal characters of $\mathrm{ZnO}$, as well as its crystal structure and defect properties. ${ }^{4-8}$ As a standard second-order nonlinear optical process, second harmonic generation (SHG) has been increasingly studied in $\mathrm{ZnO}$ bulk crystals and nanostructures. ${ }^{9-13}$ However, to the best of our knowledge, there have been no reports about the study of nonlinear optical properties and X-ray diffraction (XRD) rocking curves of ionimplanted $\mathrm{ZnO}$ single crystals. In this article, we fill in this gap by presenting an interesting investigation on nonlinear optical properties of $\mathrm{ZnO}$ single crystals implanted by $\mathrm{He}, \mathrm{Cu}$, and $\mathrm{Zn}$ ions. Both He- and $\mathrm{Zn}$-implanted samples show some improvement in SHG efficiency while the Cu-implanted one does not show observable change compared with the control sample. Xray rocking curve measurements reveal that formation of quasiinterfaces in He- and Zn-implanted samples is responsible for the observed SHG improvement in these two samples.

\footnotetext{
a) Author to whom correspondence should be addressed. Electronic mail: sjxu@hku.hk.

b) Present address: Laboratoire "Materiaux et Phenomenes Quantiques," Universite Paris Diderot-Paris 7, 75013 Paris, France.
}

\section{EXPERIMENTAL}

High-quality $\mathrm{ZnO}$ single crystals (Cermet, Inc.) with a thickness of $0.5 \mathrm{~mm}$ were used in this study. They were synthesized by the melt grown method and polished on one face which is terminated by Zn. Their optical and crystalline quality were well confirmed by low temperature photoluminescence spectra which exhibit very intensive narrow bound exciton emissions and almost no impurity/defect related visible emission (not shown here). Three different ions, namely, $\mathrm{He}, \mathrm{Cu}$, and $\mathrm{Zn}$ were implanted into $\mathrm{ZnO}$ single crystals at room temperature, 300 and $300^{\circ} \mathrm{C}$, respectively. An equal fluence of $10^{14} \mathrm{~cm}^{-2}$ was used for all the three ions. Ion energies were 100,100 , and $500 \mathrm{keV}$ for $\mathrm{He}, \mathrm{Cu}$, and $\mathrm{Zn}$, respectively. The ion concentration profile inside $\mathrm{ZnO}$ substrates was calculated by the Monte Carlo program TRIM (Transport of Ions in Matter), ${ }^{14}$ and the results were shown in Fig. 1. As can be seen from Fig. 1, the penetration depth of He ions is much deeper than that of $\mathrm{Cu}$ ions for the same ion energy. Thermal annealing treatment was performed on the implanted samples in the atmosphere of argon gas at $900^{\circ} \mathrm{C}$ for $30 \mathrm{~min}$ for further investigations. For SHG measurements, a femtosecond laser with a pulse width of $\sim 80 \mathrm{fs}$ and a repetition rate of $82 \mathrm{MHz}$, produced from a self-mode-locked Ti:sapphire oscillator (Tsunami) pumped by a $10 \mathrm{~W}$ solid-state laser (Millennia), was used as the excitation source. Emission signals from the samples were collected by a pair of lenses, analyzed by Acton SP305 monochromator, and detected with a Hamamatsu photomultiplier tube (R928). A standard lock-in amplification technique was used to improve the signal to noise ratio. A halfwave plate was used to rotate the polarization direction of the excitation beam. All SHG measurements were performed at room temperature. X-ray diffraction $2 \theta$ scanning and rocking 


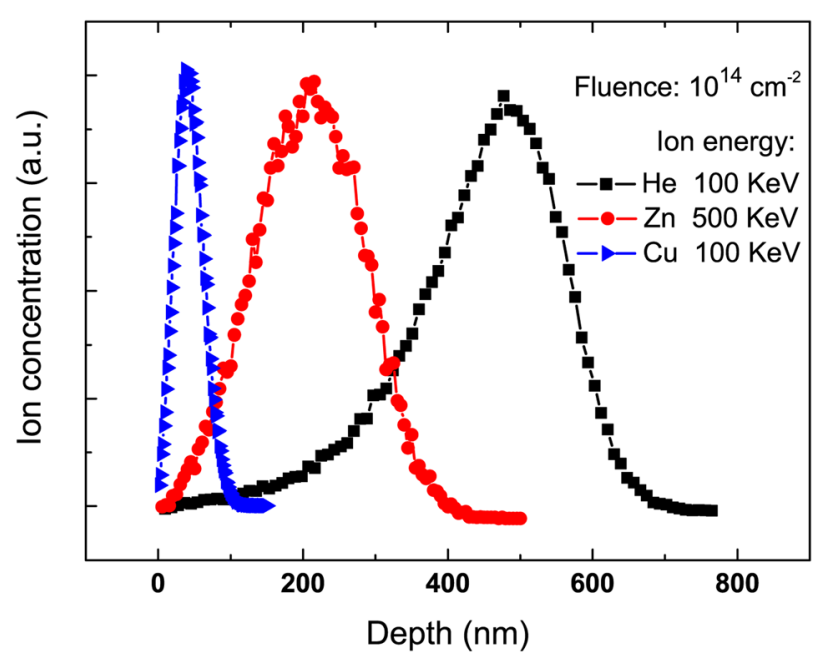

FIG. 1. (Color online) Ion concentration distributions vs depth calculated by TRIM. Rectangles + line for $\mathrm{He}$ ions in $\mathrm{ZnO}$, circulars + line for $\mathrm{Zn}$ ions while triangles + line for $\mathrm{Cu}$ ions.

curves of the samples were conducted on an X-ray diffractometer (Bruker, AXS D8 Discover) at room temperature.

For SHG measurements, the incident angle of the excitation beam was about $26^{\circ}$. The collection direction was normal to the $\mathrm{ZnO}$ sample surface (parallel to the c-axis of the sample). The excitation beam was originally linear polarized in the vertical direction. Because of the relatively strong band-edge emission under two-photon excitation, ${ }^{10}$ the wavelength of the excitation beam was chosen as $\sim 840 \mathrm{~nm}$. During the SHG measurements, the power of the excitation beam was kept as a constant $(\sim 400 \mathrm{~mW})$ by using a neutral metallic filter. Under these conditions, both the band-edge emission and SHG signal were observed, as shown in Fig. 2.

\section{RESULTS AND THEORETICAL ANALYSIS}

Figure 2(a) shows the results for pre-annealed samples including one non-implanted sample, whereas Fig. 2(b) depicts the results for post-annealed ones. The spectra were normalized at the peak of the band-edge emission for a better comparison of the SHG signals. A double-Gaussian-function fitting was adopted to fit the band-edge emission $(\sim 396 \mathrm{~nm})$ and SHG ( 420 nm), and the same method was used in determining the relative intensity of the two peaks in excitation polarization dependence measurements. By taking and comparing the ratio of the integrated intensity of SHG signal to that of the band-edge emission between implanted and non-implanted samples, we can know that the ratios of preannealed sample implanted with $\mathrm{He}^{+}$increases by $16 \%$ and by $17 \%$ after annealing. Increment of the ratio of $\mathrm{Zn}$ implanted sample was $23 \%$. However, the increment drops to $12 \%$ after experiencing post-implantation annealing. It seems that $\mathrm{Cu}$ implantation did not have significant influence on SHG efficiency of $\mathrm{ZnO}$. As seen below, theoretical analysis and arguments on the experimental results were given.

Compared with the non-implanted sample, there is an additional quasi-interface forming at a short distance from the sample surface in the ion-implanted $\mathrm{ZnO}$ samples. Within this quasi-interface region, there are higher concen-

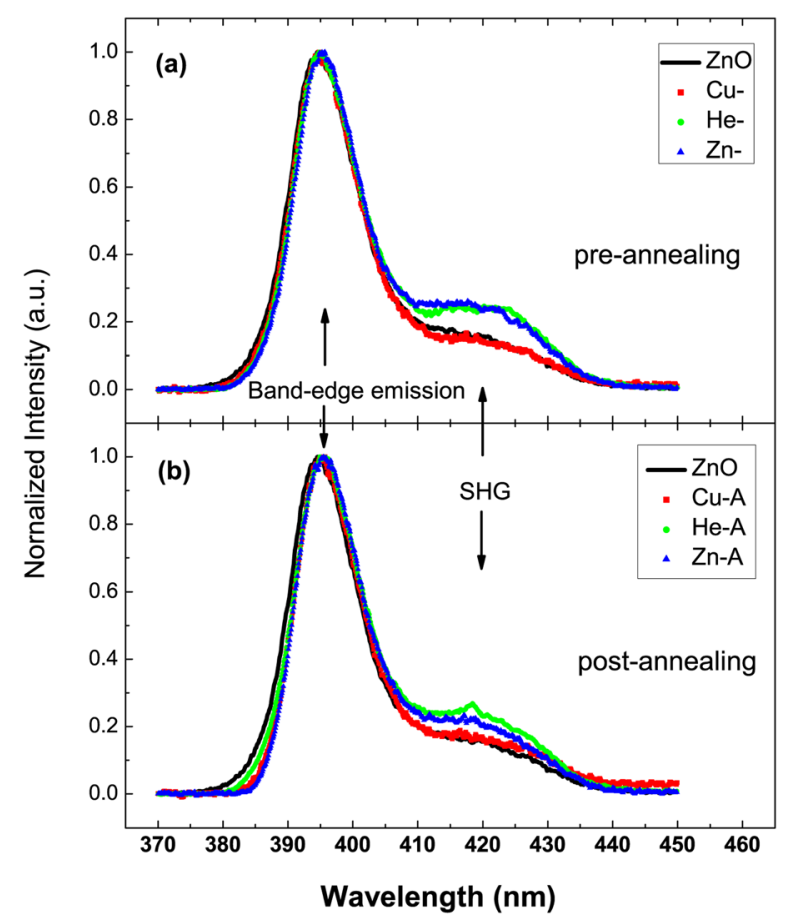

FIG. 2. (Color online) Emission spectra from pre-annealed samples (a) and post-annealed samples (b), compared with the as-grown one (solid line). In (a) and (b), solid squares for $\mathrm{Cu}$-implanted sample, solid circles for $\mathrm{He}$ implanted and solid triangles for Zn-implanted. For clear comparison, the band-edge emissions of all the samples were renormalized.

trations of implanted ions and induced disorders. Such quasiinterface shall be responsible for the observed improvement in SHG efficiency of the implanted samples. Treating the effect of the quasi-interface inside the crystal as "a thin film" 15 we can develop a simple interpretation to the experimental results. For the non-implanted sample, SHG comes from two parts and can be expressed as ${ }^{16}$

$$
I_{S H G}=I_{S H G}^{B}+I_{S H G}^{S},
$$

where $I_{S H G}^{B}$ represents the contribution from the bulk, and $I_{S H G}^{S}$ the contribution from the surface. For ion-implanted samples, one additional term should be added to the righthand side of Eq. (1), $I_{S H G}^{F}$, taking into account the contribution from the thin film. For a film thickness that is comparable with the wavelength of the fundamental beam, the SHG from the film is proportional to the square of the film thickness and the effective second-order susceptibility, ${ }^{17}$

$$
I_{S H G}^{F} \propto d^{2} \cdot\left|\chi_{e f f}^{(2)}\right|^{2}
$$

where $d$ denotes the thickness of the film and $\chi_{\text {eff }}^{(2)}$ the effective second-order susceptibility. Simply supposing that $\chi_{\text {eff }}^{(2)}$ is the same for the thin films induced by different ionimplantations, the main parameter affecting SHG would be the film thickness. Considering the ion concentration profiles, we have two approaches to evaluate the film thickness: (I) the distance from the crystal surface to the ion concentration peak and (II) the full width at half maximum of the ion concentration profile. For method (I), the thicknesses were estimated as 37.5, 215.0, and $477.0 \mathrm{~nm}$ for $\mathrm{Cu}-, \mathrm{Zn}-$, and 
He-implanted samples, respectively; while for method (II), the thickness as 48.0, 185.0, and $207.0 \mathrm{~nm}$ for $\mathrm{Cu}-, \mathrm{Zn}-$, and He-implanted samples, respectively. From the experimental results, method (II) seems more reasonable as the improvement of SHG efficiency of $\mathrm{Zn}$ - and He-implanted samples is more noticeable than that of the $\mathrm{Cu}$-implanted sample.

To further investigate the influence of ion-implantation on SHG of $\mathrm{ZnO}$ single crystals, the excitation polarization dependence of SHG and the band-edge emission from the preannealed samples with and without ion-implantation was measured. Figure 3(a) depicts the results from the nonimplanted sample, while Fig. 3(b) for the Zn-implanted sample. Results (not shown here) measured from He-implanted sample were similar to those from $\mathrm{Zn}$-implanted sample. Solid triangles represent the SHG signal while solid circles for the band-edge emission. Again, the polarization dependence measurements show additional evidence for the improvement of SHG in the ion-implanted samples. It is known that SHG intensity is proportional to the square of second-order polarization induced by the fundamental beam, ${ }^{16,18}$

$$
I_{S H G} \propto\left[P^{(2)}\left(\omega=2 \omega_{0}\right)\right]^{2},
$$

where $P^{(2)}\left(\omega=2 \omega_{0}\right)=\chi_{i j k}^{(2)} E_{j} E_{k}, \chi_{i j k}^{(2)}$ is the second-order susceptibility of the crystal. Since wurtzite $\mathrm{ZnO}$ crystal belongs to the $C_{6 v}$ (or $6 \mathrm{~mm}$ ) point group symmetry, and under Kleinman symmetry conditions, its two nonzero second-order susceptibility elements are $\chi_{z z z}^{(2)}=-14.31 \mathrm{pm} / \mathrm{V}$, and $\chi_{z x x}^{(2)}=1.36 \mathrm{pm} / \mathrm{V} .{ }^{19}$ Considering that the incident angle is $\sim 26^{\circ}$ in the present study, the second-order susceptibility as

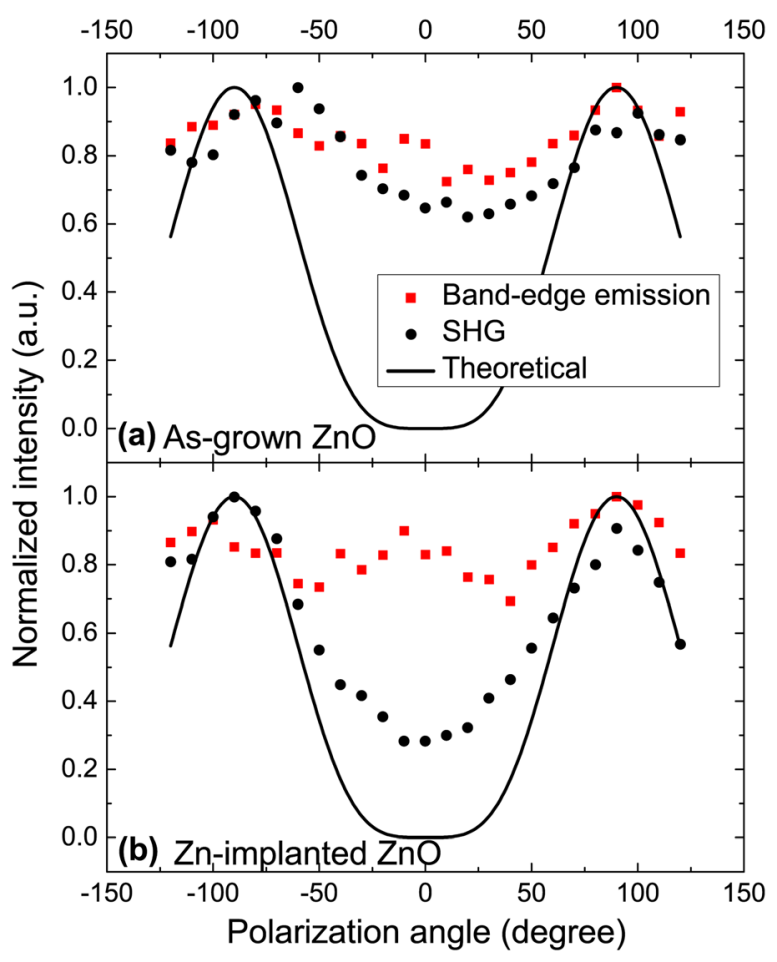

FIG. 3. (Color online) Measured dependence of integrated intensity of SHG (solid circles) and band-edge emission (solid squares) on polarization of the excitation beam: (a) As-grown $\mathrm{ZnO}$; (b) $\mathrm{Zn}$-implanted $\mathrm{ZnO}$. The solid line represents a theoretical polarization dependence of the standard SHG signal. a function of the linear polarization angle $\varphi$ of the incident fundamental beam can be derived as

$$
P^{(2)}\left(\omega=2 \omega_{0}\right) \propto 2.73 \sin ^{2} \varphi
$$

Calculated dependence (solid line) of an ideal SHG signal on the polarization of incident fundamental beams using Eqs. (3) and (4) is shown in Fig. 3. Compared with the nonimplanted sample, relatively stronger SHG dependence on the excitation polarization direction in $\mathrm{Zn}$ - and He-implanted samples were observed, indicating the enhanced SHG efficiency in these ion-implanted samples.

The possible influence of thermal annealing on the SHG in the ion-implanted samples was also investigated as thermal treatment can have significant impact on disorder recovery. The results are shown in Fig. 2(b). As expected, the SHG signals from the annealed ion-implanted samples exhibits some degradation possibly due to disorder improvement and recrystallization. ${ }^{20}$ As for the $\mathrm{Cu}$-implanted sample, no noticeable change of SHG efficiency was observed before and after thermal annealing due to the shallow and narrow copper ion distribution in $\mathrm{ZnO}$. The XRD patterns shown and discussed below from the samples give interesting and positive information, which is consistent with the SHG observations.

Figure 4 shows measured XRD rocking curves of the $\mathrm{ZnO}$ samples and the inset of Fig. 4(a) depicts the XRD $2 \theta$ scanning pattern of the as-grown sample. Within the $2 \theta$ scanning range $\left(30^{\circ}-70^{\circ}\right)$, only one peak around $34.42^{\circ}$ was observed for all the samples, which was assigned to the [002] peak from hexagonal ZnO (PDF pattern: 36-1451). However, a remarkable difference between the rocking curves of the samples was interestingly observed. As seen from Fig. 4, for pre-annealed $\mathrm{Cu}$-implanted $\mathrm{ZnO}$, its rocking curve is almost the same as the as-grown one. This is consistent with the SHG result that no improvement of SHG was observed from the $\mathrm{Cu}$-implanted one. In contrast to the case of $\mathrm{Cu}$-implanted sample, the

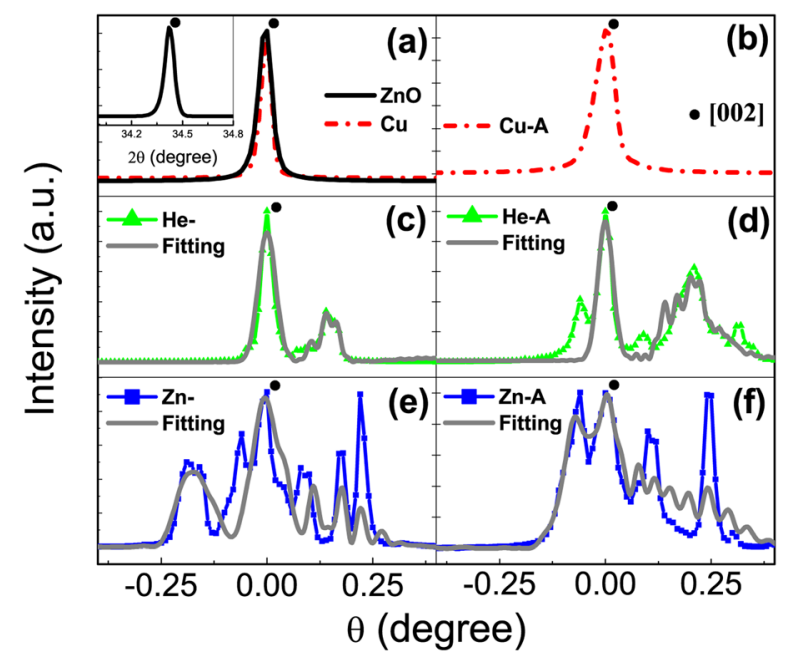

FIG. 4. (Color online) XRD rocking curves of the $\mathrm{ZnO}$ samples: (a) and (b) for $\mathrm{Cu}$-implanted $\mathrm{ZnO}$ before and after thermal annealing, respectively; (c) and (d) for He-implanted; (e) and (f) for Zn-implanted. The solid line in Fig. 4(a) is for the as-grown ZnO. The inset in Fig. 4(a) shows XRD $2 \theta$ scanning pattern of the as-grown $\mathrm{ZnO}$. The solid lines (gray color) in (c), (d), (e), and (f) are the simulation results for the corresponding XRD rocking curves, respectively. 
rocking curves of $\mathrm{He}$ - and $\mathrm{Zn}$-implanted samples show several satellite peaks, indicating the formation of coarse-grains or quasi-interfaces in these ion-implanted samples. Generally, XRD rocking curves of multiple layered heterostructures like superlattices and even ion-implanted semiconductors exhibit several satellite peaks. ${ }^{21-23}$ The formation of quasi-interfaces inside ion-implanted crystals will certainly cause some improvement of the SHG efficiency due to the contribution of these symmetry breaking interfaces to the nonlinear coefficient. $^{13,16,24}$ The rocking curves of annealed ion-implanted samples are also consistent with the SHG results.

A kinematical model ${ }^{25}$ developed from the uniform single crystal dynamical theory ${ }^{26}$ was adopted to explain the formation of quasi-interfaces by fitting the XRD rocking curves. The model includes depth-dependent strain and spherically symmetric Gaussian distribution of randomly displaced atoms. Depth-dependent strain distributions are represented by a set of independently but coherently diffracting laminae oriented parallel to the surface. Each lamina contains many unit cells and has uniform strain. Random displacements/damages are treated through their effect on the mean structure factor in each lamina.

The plane-wave dynamical theory predicts that for unit electric field amplitude incident on the surface of an isolated, uniform, non-absorbing, single crystal plate, the diffracted amplitude at the same surface is ${ }^{26}$

$$
E_{D}=e^{-i 2 \pi\left(\mathbf{K}_{\mathbf{0}}^{\mathbf{e}}+\mathbf{B}_{\mathbf{H}}\right) \cdot \mathbf{r}} D_{D}
$$

where $\mathbf{K}_{\mathbf{0}}^{\mathbf{e}}$ is the incident external wavevector, $\left|\mathbf{K}_{\mathbf{0}}^{\mathbf{e}}\right|$ $=1 / \lambda, \mathbf{B}_{\mathbf{H}}$ is the reciprocal lattice vector, $\mathbf{r}$ is the vector from origin (chosen on the surface). If the plate thickness and/or the structure factor are sufficiently small, $D_{D}$ can be written as

$$
D_{K}=i \frac{F_{H}}{\left|F_{H}\right|} \sqrt{|b|} e^{-i A Y} \frac{\sin (A Y)}{A Y},
$$

where $F_{H}$ is the structure factor, $b=\gamma_{0} / \gamma_{\mathrm{H}} ; \gamma_{0}, \gamma_{\mathrm{H}}$ are direction cosines of incident and diffracted wavevectors, respectively, from the inward normal to the surface,

$$
A=r_{e} \frac{\lambda\left|F_{H}\right|}{V} \frac{t}{\sqrt{\left|\gamma_{0} \gamma_{H}\right|}},
$$

where $r_{e}=e^{2} / m c^{2}$ is the classical electron radius, $V$ is the volume of unit cell, $t$ is the plate thickness,

$$
\begin{aligned}
& Y=\frac{\psi_{0}(1-b)+b \alpha}{2 \sqrt{|b|}\left|\psi_{H}\right|}, \\
& \psi_{0, H}=-r_{e} \frac{\lambda^{2}}{\pi} \frac{F_{0, H}}{V}, \\
& \alpha=-2 \Delta \theta \sin 2 \theta_{B}, \\
& \Delta \theta=\theta-\theta_{B}, \\
& \theta_{B} \text { is the Bragg angle. }
\end{aligned}
$$

Equations (6)-(8) are valid only for $\sigma$-polarization. For $\pi$-polarization $F_{H}$ is replaced by $F_{H} \cos 2 \theta_{B}$. If the lattice is strained in a direction perpendicular to the sample surface, the corresponding change in Eqs. (8) is

$$
\Delta \theta \rightarrow \Delta \theta+\epsilon^{\perp}\left[\left|\gamma_{H}\right| \sqrt{1-\gamma_{H}^{2}}+\gamma_{H}^{2} \tan \theta_{B}\right] .
$$

Here $\epsilon^{\perp}$ is the strain and correction includes changes in the direction and magnitude of the reciprocal lattice vector.

For ion-implanted crystals, a significant fraction of atoms may be displaced from lattice positions, which may lead to the formation of quasi-interfaces in the implanted region. The statistical distribution of displacements $\Delta \mathbf{r}_{\mathbf{j}}$ away from lattice $\mathbf{j}$ is described by a function $\rho\left(\Delta \mathbf{r}_{\mathbf{j}}\right)$. Such a distribution will result in a mean structure factor

$$
\left\langle F_{H}\right\rangle=\sum_{j} f_{j} \int d^{3} r \rho\left(\Delta \mathbf{r}_{\mathbf{j}}\right) e^{-i 2 \pi \mathbf{B}_{\mathbf{H}} \cdot\left(\mathbf{r}_{\mathbf{j}}+\Delta \mathbf{r}_{\mathbf{j}}\right)},
$$

where $f_{j}$ is the atomic scattering factor for site $j$, located at $\mathbf{r}_{\mathbf{j}}$ in an undamaged crystal. If the same spherically symmetric Gaussian form is assumed for all sites, the mean structure factor becomes

$$
\left\langle F_{H}\right\rangle=\exp \left(-\frac{8 \pi^{2}}{\lambda^{2}} \sin ^{2} \theta_{B} U^{2}\right) F_{H}^{0},
$$

where $F_{H}^{0}$ corresponds to undamaged crystal and $U$ is the standard deviation of displacements.

Strain and damage distributions are represented by a set of discrete laminae oriented parallel to the surface. Each lamina contains a large number of unit cells, but is sufficiently thin so that extinction and normal absorption within the lamina are negligible. Each lamina has its own uniform strain $\epsilon^{\perp}$ and random displacement standard deviation $U$. Dynamical interactions among different laminae are neglected, as is the effect of extinction on the incident wave. The total diffracted amplitude is then the sum of coherently interfering functions of the type shown in Eqs. (6), adjusted for phase lags and normal absorption during traversal through the crystal. Although usually extinction is stronger than normal absorption, for depth-dependent strain distributions the latter can be more important, which is the case of ion-implanted crystals. With these considerations, the total amplitude from $N$ laminae can be written as

$$
E_{N}=i \frac{F_{H}}{\left|F_{H}\right|} \sqrt{|b|} \sum_{j=1}^{N} a_{j} e^{-i\left(A_{j} Y_{j}+\phi_{j}\right)} \frac{\sin \left(A_{j} Y_{j}\right)}{Y_{j}}
$$

where

$$
\begin{aligned}
& a_{j}=\exp \left[-\mu \frac{\gamma_{0}+\left|\gamma_{H}\right|}{2\left|\gamma_{0} \gamma_{H}\right|} \sum_{l=j+1}^{N} t_{l}\right] ; \quad a_{N}=1, \\
& \mu=\frac{2 \lambda}{V} r_{e} \operatorname{Im}\left(F_{0}\right), \\
& t_{l}=\text { thickness of lamina } l, \\
& \phi_{j}=2 \sum_{l=1}^{j-1} A_{l} Y_{l} ; \quad \phi_{1}=0,
\end{aligned}
$$




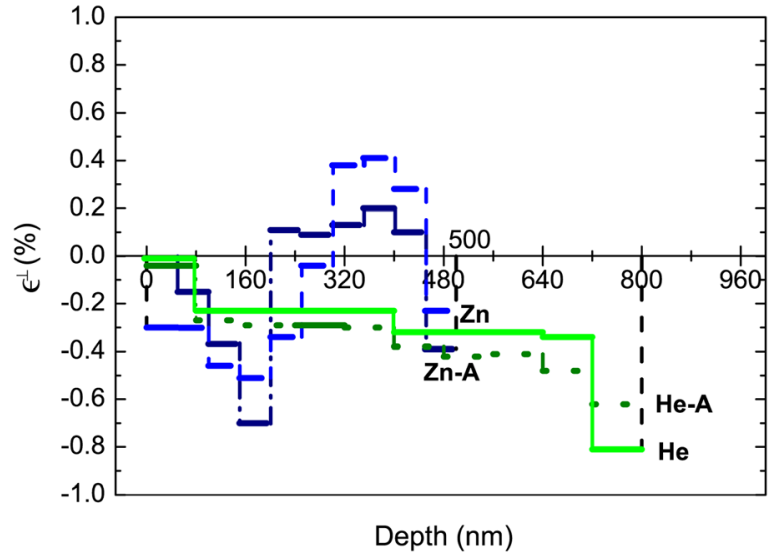

FIG. 5. (Color online) Strain profiles used for simulation of the XRD rocking curves of He- and Zn-implanted samples. He-implanted samples: preannealed (solid lines, marked by He); post-annealed (dotted lines, marked by He-A). Zn-implanted ones: pre-annealed (dashed lines, marked by Zn); post-annealed (dashed-dotted lines, marked by Zn-A).

and the previously defined variables $\mathrm{Y}$ and $\mathrm{A}$ are now subscripted to indicate dependence on strain and damage. In addition, since Eqs. (12) will be used to determine strain relative to virgin crystal, the refraction correction $\psi_{0}(1-b)$ in the definition of $\mathrm{Y}$ in Eqs. (8) is neglected. The total calculated intensity would be the sum of layer and substrate intensities, which is

$$
R_{T}=\frac{1+\cos ^{4} 2 \theta_{B}}{1+\cos ^{2} 2 \theta_{B}} \frac{\left|\gamma_{H}\right|}{\gamma_{H}} E_{N} E_{N}^{*}+R_{S}
$$

where the first factor represents the relative abundance of $\sigma$ - and $\pi$-polarization in double crystal diffractometry, $\left|\gamma_{\mathrm{H}}\right| / \gamma_{0}$ relates intensity to power, and $R s$ is the substrate contribution which is taken as a Gaussian profile.

For fitting the XRD rocking curves, the thickness of each lamina is the same and chosen to be in the range of tens $\mathrm{nm}$ (80 nm for He-implanted samples; $50 \mathrm{~nm}$ for Zn-implanted samples). The number of laminae ( $N=10$ in all simulations) is determined by the lamina thickness and the total thickness of the ion-implanted region which is indicated by ion concentration profile calculated by the Monte Carlo program TRIM. The simulation results were shown in gray lines for the corresponding XRD rocking curves in Figs. 4(c), 4(d), 4(e), and 4(f), respectively. The strain values of each lamina along the c-axis of the crystal are given from the fitting parameters. A negative strain profile (compressive strain) was found for Heimplanted samples, both pre- and post-annealed ones. For Znimplanted samples, both positive and negative strains were revealed for the best fitting results, which were similar to the simulation results of As-implanted silicon. ${ }^{23}$ Figure 5 shows the strain profile of all four samples extracted from the fitting results. The strain in the ion-implanted region was induced by substitutions and interstitial of original lattice sites by implanted ions and the vacancy-interstitial pairs caused by the collision of foreign ions with atoms of the target. ${ }^{27}$ It seems that $\mathrm{Zn}$ ions with high ion energy had more prominent effects of introducing structural defects. The simulation results of XRD rocking curves of both He- and $\mathrm{Zn}$-implanted samples confirm that an overall strain layer was formed near the surface of the samples, which would cause the improvement of SHG efficiency.

\section{CONCLUSIONS}

In summary, SHG and XRD rocking curves of $\mathrm{ZnO}$ single crystals implanted by $\mathrm{He}, \mathrm{Cu}$, and $\mathrm{Zn}$ were experimentally investigated. It has been shown that ion-implantation can lead to some improvement of SHG efficiency if quasiinterfaces form in implanted samples. The polarization dependence of SHG signals on the excitation light and XRD results also support this conclusion.

\section{ACKNOWLEDGMENTS}

This work was financially supported by HK RGC-GRF Grants (No. HKU 7056/06P) and Joint Research Fund for Overseas Chinese, Hong Kong and Macau Scientists of NSFC (No. 61028012). This work was also partially supported by University Grants Committee Areas of Excellence Scheme of the Hong Kong Special Administrative Region, China (Project No. [AoE/P-03/08]).

${ }^{1}$ Ü. Özgür, Y. I. Alivov, C. Liu, A. Teke, M. A. Reshchikov, S. Dogan, V. Avrutin, S. J. Cho, and H. Morkoç, J. Appl. Phys. 98, 041301 (2005).

${ }^{2}$ C. Klingshirn, J. Fallert, H. Zhou, J. Sartor, C. Thiele, F. Maier-Flaig, D. Schneider, and H. Kalt, Phys. Status Solidi B 247, 1424 (2010).

${ }^{3}$ S. J. Pearton, D. P. Norton, K. Ip, Y. W. Heo, and T. Steiner, Prog. Mater. Sci. 50, 293 (2005).

${ }^{4}$ S. O. Kucheyev, P. N. K. Deenapanray, C. Jagadish, J. S. Williams, M. Yano, K. Koike, S. Sasa, M. Inoue, and K. Ogata, Appl. Phys. Lett. 81, 3350 (2002).

${ }^{5}$ S. O. Kucheyev, C. Jagadish, J. S. Williams, P. N. K. Deenapanray, M. Yano, K. Koike, S. Sasa, M. Inoue, and K. Ogata, J. Appl. Phys. 93, 2972 (2003).

${ }^{6}$ V. A. Coleman, H. H. Tan, C. Jagadish, S. O. Kucheyev, and J. Zou, Appl. Phys. Lett. 87, 231912 (2005).

${ }^{7}$ A. Y. Azarov, S. O. Kucheyev, A. I. Titov, and P. A. Karaseov, J. Appl. Phys. 102, 083547 (2007).

${ }^{8}$ C. Y. Zhu, C. C. Ling, G. Brauer, W. Anwand, and W. Skorupa, Microelectron. J. 40, 286 (2009).

${ }^{9}$ G. Wang, G. T. Kiehne, G. K. L. Wong, J. B. Ketterson, X. Liu, and R. P. H. Chang, Appl. Phys. Lett. 80, 401 (2002).

${ }^{10}$ D. C. Dai, S. J. Xu, S. L. Shi, M. H. Xie, and C. M. Che, IEEE Photonics Technol. Lett. 18, 1533 (2006).

${ }^{11}$ T. Voss, I. Kudyk, L. Wischmeier, and J. Gutowski, Phys. Status Solidi B 246, 311 (2009).

${ }^{12}$ B. P. Mehl, R. L. House, A. Uppal, A. J. Reams, C. Zhang, J. R. Kirschbrown, and J. M. Papanikolas, J. Phys. Chem. A 114, 1241 (2010).

${ }^{13}$ S. L. Shi. S. J. Xu, Z.-X. Xu, V. A. L. Roy, and C.-M. Che, Chem. Phys. Lett. 506, 226 (2011).

${ }^{14}$ J. F. Ziegler, see http://www.srim.org for SRIM \& TRIM.

${ }^{15}$ J. M. Mayer, L. Eriksson, and J. A. Davies, Ion Implantation in Semiconductors (Academic, New York, 1970).

${ }^{16}$ Y. R. Shen, The Principles of Nonlinear Optics (Academic, New York, 1993).

${ }^{17}$ N. Bloembergen and P. S. Pershan, Phys. Rev. 128, 606 (1962).

${ }^{18}$ H. Yang, S. J. Xu, Q. Li, and J. Zhang, Appl. Phys. Lett. 88, 161113 (2006).

${ }^{19}$ G. Wang, G. K. L. Wong, and J. B. Ketterson, Appl. Opt. 40, 5436 (2001).

${ }^{20}$ H. H. Tan, J. S. Williams, J. Zou, D. J. H. Cockayne, S. J. Pearton, J. C. Zolper, and R. A. Stall, Appl. Phys. Lett. 72, 1190 (1998).

${ }^{21}$ D. Pan, Y. P. Zeng, J. Wu, H. M. Wang, C. H. Chang, J. M. Li, and M. Y. Kong, Appl. Phys. Lett. 70, 2440 (1997).

${ }^{22}$ S. J. Xu, H. Wang, Q. Li, M. H. Xie, X. C. Wang, W. J. Fan, and S. L. Feng, Appl. Phys. Lett. 77, 2130 (2000).

${ }^{23}$ M. Nemiroff and V. S. Speriosu, J. Appl. Phys. 58, 3735 (1985). 
${ }^{24}$ C. C. Zheng, S. J. Xu, J. Q. Ning, S. F. Zhang, J. Y. Wang, C. M. Che, and J. H. Hao, J. Appl. Phys. 109, 013528 (2011).

${ }^{25}$ V. S. Speriosu, J. Appl. Phys. 52, 6094 (1981).
${ }^{26} \mathrm{~W}$. H. Zachariasen, Theory of X-Ray Diffraction in Crystals (Wiley, New York, 1945).

${ }^{27}$ A. Pesek, Appl. Phys. A A58, 141 (1994). 